\title{
La croix et l'ordre : le clergé et la censure de l'imprimé au Québec
}

\author{
The crucifix and order: the clergy and print censorship in \\ Québec
}

\section{La cruz y el orden: el clero y la censura de los impresos en la provincia de Quebec}

\section{Pierre Hébert}

Volume 41, numéro 1, janvier-mars 1995

URI : https://id.erudit.org/iderudit/1033349ar

DOI : https://doi.org/10.7202/1033349ar

Aller au sommaire du numéro

Éditeur(s)

Association pour l'avancement des sciences et des techniques de la documentation (ASTED)

ISSN

0315-2340 (imprimé)

2291-8949 (numérique)

Découvrir la revue

Citer cet article

Hébert, P. (1995). La croix et l'ordre : le clergé et la censure de l'imprimé au Québec. Documentation et bibliothèques, 41(1), 21-29.

https://doi.org/10.7202/1033349ar
Résumé de l'article

En notre fin de siècle, les jeunes spécialistes de la documentation ont peine à imaginer la place que la censure a occupée dans notre histoire intellectuelle. Cet article situe l'histoire de la censure ecclésiastique et définit son importance à travers les siècles. Il pose aussi la question du poids de cette censure dans le vie culturelle du Québec et suggère de nouvelles pistes de recherche pour mieux comprendre le sens de son évolution.
Tous droits réservés (c) Association pour l'avancement des sciences et des techniques de la documentation (ASTED), 1995
Ce document est protégé par la loi sur le droit d'auteur. L'utilisation des services d’Érudit (y compris la reproduction) est assujettie à sa politique d'utilisation que vous pouvez consulter en ligne. 


\title{
La croix et l'ordre: le clergé et la censure de l'imprimé au Québec
}

\author{
Pierre Hébert \\ Université de Sherbrooke
}

En notre fin de siècle, les jeunes spécialistes de la documentation ont peine à imaginer la place que la censure a occupée dans notre histoire intellectuelle. Cet article situe l'histoire de la censure ecclésiastique et définit son importance à travers les siècles. II pose aussi la question du poids de cette censure dans le vie culturelle du Québec et suggère de nouvelles pistes de recherche pourmieux comprendre le sens de son évolution.

\section{The crucifix and order: the clergy and print censorship in Québec}

As the century comes to a close, young information specialists are having a hard time imagining the effects of censorship on our intellectual history. This article describes the history of ecclesiastical censorship and its importance over time. It also addresses the impact censorship had on the cultural life of Québec and suggests how new research can lead to a better understanding of its evolution.

\section{La cruz y el orden: el clero y la censura de los impresos en la provincia de Quebec}

En este fin de siglo, los jóvenes especialistas de la documentación tienen pena a imaginar el lugar que la censura ha occupado en nuestra historia intelectual. Este artículo sitúa la historia de la censura eclesiástica y define su importancia a través de los siglos. Plantea también la cuestión de la presión de esta censura en la vida cultural de la provincia de Quebec y sugiere nuevas pistas de investigación para comprender mejor el sentido de su evolución.
J'ouvrirai cet article par une anecdote récente mais, surtout, révélatrice. $\grave{A}$ l'occasion d'un cours de $2^{\mathrm{e}}$ et de $3^{\mathrm{e}}$ cycles sur la censure religieuse, j'ai cru opportun de parler des catéchismes dans la formation de la pensée québécoise. Après quelques minutes d'explications, je me suis vite aperçu que la plupart de mes étudiantes et étudiants n'avaient aucune idée de ce dont je parlais: ils n'avaient jamais vu un catéchisme de leur vie et ce mot, pour eux, renvoyait plutôt à catéchèse. Je m'adressais à des gens qui, pour la plupart, avaient trente ans ou moins, à des «enfants de Vatican II», pour tout dire.

Comme il leur était alors difficile d'imaginer l'omniprésence et l'omnipotence du clergé dans la vie québécoise aux $19^{e}$ et $20^{e}$ siècles, du moins jusque vers 1950 ! Et pourtant, leurs aînés ont été victimes ou témoins du contrôle religieux à l'endroit de toute parole dissidente. Car il est bien connu que, pendant plus d'un siècle (disons, grossièrement, entre 1840 et 1950), le clergé a exercé un contrôle sévère, voire une répression à l'endroit de toute manifestation hétérodoxe, particulièrement dans le domaine qui m'intéresse ici, c'est-à-dire la littérature au sens large, incluant livres, journaux et représentations théâtrales. On pourrait multiplier àl'enviles interdictions adressées aux propos divergents; cette activité censoriale a marqué l'histoire du Québec et, plus particulièrement, son évolution littéraire.

Pourtant, malgré l'importance reconnue de ce fait, il n'existe encore aujourd'hui aucune histoire de la censure ecclésiastique au Québec ${ }^{1}$. Nous disposons d'études (le plus souvent des mémoires et des thèses) sur certains cas devenus classiques: Canada-Revue, Marie Calumet, Le Débutant, etc. D'autres pans sont encore peu connus: les frasques de l'abbé Pelletier sur la question des classiques, la mise à l'index du Clergé canadien, sa mission, son oeuvre de Laurent Olivier David, entre autres.

Je me propose, dans cet article, d'atteindre deux buts. En premier lieu, je vise à donner une vision d'ensemble de l'histoire de la censure ecclésiastique au Québec, des origines jusque vers 1940. J'aurais pu aborder cet objet selon les angles de la production, de la diffusion et de la consommation; mais il m'a semblé plus opportun, à ce stade, d'obéir à l'impératif chronologique, à l'intérieur duquel je proposerai néanmoins une certaine périodisation.

En second lieu, j'assortirai ces propos du plus grand nombre de références possible, afin de dresser l'état des recherches sur la question. L'on trouvera donc ici, en plus d'un parcours historique, une bibliographie disséminée dans le texte, surles rapports entre le clergé et la censure.

L'ampleur du sujet m'obligera en outre à des raccourcis: j'en appelle à l'indulgence du lecteur jusqu'à la parution d'une étude plus fouillée sur cette question ${ }^{2}$.

\section{Objet et hypothèses de périodisation}

Qu'est-ce quela censure? La réponse donnée à cette question circonscrit du même coup l'objet de cet article. Par censure $^{3}$, j'entendrai toute forme de contrôle de parole divergente, que ce contrôle soit a priori ou a posteriori. L'on connaîtl'aspect spectaculaire de la censure 
a posteriori: condamnation, interdiction, autodafé. Par contre, la censure a priori n'en est pas moins efficace, voire plus redoutable. Imbert (1983, 154-157) propose trois niveaux de censure: primaire, où l'on tronque l'oeuvre; secondaire, c'està-dire l'autocensure, et enfin tertiaire, où il. n'est pas permis «à l'utilisateur de penser, de voir, de saisir, de réfléchir, de projeter, de créer en dehors de certaines limites ramenant chacun aux a priori fondamentaux». II semble ainsi nécessaire de tenir compte de toutes ces formes comme diverses facettes de la censure. Quant à l'objet matériel, il sera constitué ici de l'imprimé et, avant le 19e siècle, des représentations théâtrales, puisque cellesci, avant l'introduction de l'imprimerie en 1764 , représentent le principal moyen de "communication littéraire» qui a inquiété le clergé.

La périodisation représente par contre une opération plus délicate. Seule une étude plus poussée permettrait de valider ce qui sera avancé comme hypothèse, encore que celle-ci m'apparaisse, bien sûr, recevable.Je propose ainsi, du régime français jusqu'au milieu du $20^{\mathrm{e}}$ siècle, quatre grandes phases dans l'évolution de la censure religieuse au Québec qui correspondront aux quatre parties de cet article:

\section{La censure casuelle (1625-1800)}

L'hypothèse qui gouverne cette première phase est celle d'une censure épisodique, qui répond au cas par cas. II ne faut pas se faire l'idée d'une censure ecclésiastique trop bien organisée, sous le régime français, et durant les quatre décennies qui ont suivi la conquête anglaise.

\section{La censure impraticable (1800-1840)}

La faiblesse du pouvoir clérical et les attaques dont il sera même la victime, caractérisent ce deuxième volet. Toutefois, ces années difficiles s'avéreront aussi décisives: elles conféreront au clergé un savoiret un vouloirqui culmineront dans le pouvoir de la prochaine étape.

\section{La censure intensive-extensive I (1840-1892)}

Je pose que non seulement le pouvoir du clergé s'organise à partir de 1840 , mais que celui-ci s'articule en deux temps: intensif et extensif. La phase intensive (1840-1876) se caractérise parune censure présente è récurrente, surtout à l'endroit de l'Institut canadien et des principales tribunes libérales. La phase extensive (1876-1892) désigne les résultats de la phase intensive: la censure a été tellement bien exécutée dans la première phase que la seconde se qualifie par un contrôle a priori des paroles divergentes, àl'exception de la querelle au sujet de l'Université Laval et de sa succursale montréalaise.

\section{La censure intensive-extensive II (1892-1940)}

Un renouveau d'anticléricalisme, à la fin du $19^{\mathrm{e}}$ siècle, entraîne un mouvement de censure intensive pendant une vingtaine d'années $\left(1892-1913^{5}\right)$; la censure extensive est ensuite assurée par l'action positive, incarnée surtout dans les mouvements d'action catholique qui naissent au début du siècle et se consolident dans la décennie suivante.

Je suivrai donc ces phases en les caractérisant par les événements qui les constituent, et en donnant le bilan de nos connaissances sur le sujet.

\section{La censure casuelle (1625-1800)}

Le premier cas connu de censure est celui de l'Anti-Coton. II s'agit d'un pamphlet contre le jésuite Pierre Coton (Bulletin des recherches historiques 1895), qui semble être notre premier autodafé. À première vue, la censure a frappé rapidement, pouvant donner ainsi l'image d'une jeune colonie fortement policée. Doit-on entériner pareille hypothèse? Durant le régime français, probablement pas, du moins si l'on suit le jugement d'Antoine Roy:

À l'encontre de ce que l'on croit d'ordinaire, une certaine indépendance intellectuelle avait toujours existé au Canada. [...] En réalité, les prêtres ne paraissaient pas avoir beaucoup gêné la libre activité des cenveaux canadiens. Grands liseurs eux-mêmes, ils ne damnaient pas pour si peu que de ne pas venir prendre chez eux le mot d'ordre. Le cas de l'Anti-Coton qui fut brûlé par la main du bourreau en 1626 reste un exemple isolé (Roy 1930, 8485).
Ce n'est toutefois pas l'opinion de JeanPaul de Lagrave $(1975,131$ p.), qui voit dans le régime français une époque très coercitive. La vérité? sans doute, comme d'habitude, entre les deux... Précisons que, comme les Jésuites sont arrivés en Nouvelle-France à l'été 1625 et que l'AntiCoton a été brûlé quatre mois après leur arrivée (selon les Relations), c'est plutôt en 1625 que ces événements auraient eu lieu.

Les esclandres de Mgr de SaintVallier appellent des réserves semblables. On connaît la théâtrophobie de l'évêque: déjà vers 1685 , il écrivait, dans son «Avis donnés au Gouverneur et à la Gouvernante sur l'obligation où ils sont de donner le bon exemple au peuple», que la fille du Gouverneur ne devait pas «paraître devant le monde comme une actrice en déclamant des vers», car "ce serait renouveler sans y penser l'usage du théâtre et de la comédie, ou autant ou plus dangereuse que le bal et la danse, et contre laquelle les désordres qui en sont arrivés autrefois ont donné lieu d'invectiver avec beaucoup de véhémence» (Mandements... (MÉQ) tome 1 171-172). Inutile de revenir ici sur cet épisode bien connu des démêlés de SaintVallier avec Frontenac et à l'interdiction que l'Évêque fera de la représentation de Tartuffe de Molière (Laflamme et Tourangeau 1979,58-73). Son Mandement au sujet des comédies, de même que l'Éclaircissement touchant la comédie du chanoine Charles de Glandelet, sont péremptoires. Venant de nommer Tartuffe, l'évêque proclame

Nous déclarons que ces sortes de spectacles et de comédies ne sont pas seulement dangereuses, mais qu'elles sont absolument mauvaises et criminelles d'elles-mêmes et qu'on ne peut $y$ assister sans péché, et comme telles nous les condamnons et faisons défenses très expresses à toutes les personnes de notre Diocèse de quelque qualité et condition qu'elles soient de s'y trouver. (MÉQ tome 1, 303)

Cette condamnation eut certes un effet néfaste sur le développement du théâtre en Nouvelle-France:«En 1699, il n'y avait plus au Collège ni déclamation, ni tragédie», écrit Antoine Roy $(1930,125)$. Toutefois, durant ce régime de censure cașuelle, il faut noter ce jugement: «C'est 
une des rares circonstances où, sous le régime français, le clergé avait eu gain de cause.» (Roy 1930, 126).

Le clergé est donc bien conscient de la menace que constituent le théâtre et l'imprimé; mais on connaît peu d'esclandre semblable à celui de «l'affaire du Tartuffe» au $18^{\mathrm{e}}$ siècle $^{6}$, à l'exception de la saisie du journal de Fleury Mesplet et de Valentin Jautard, La Gazette littéraire, pour la Ville \& District de Montréal, en juin 1779. Depuis un an, Jautard et Mesplet agitaient les cerveaux et les passions par leur journal qui, participant de l'esprit des Lumières, disséminait les idées voltairiennes, appuyait l'Académie de Montréal et, même, attaquait les "Écoliers de Montréal». Des démêlés judiciaires serviront de prétexte ultime au gouverneur Haldimand pour saisir La Gazette littéraire [..], qui venait de publier une feuille pamphlétaire intitulée «Tant pis, tant mieux». Jautard et Mesplet iront réfléchir quelques années en prison... Cette censure paraît être due au pouvoir civil; mais le clergé était intervenu privément ou publiquement sous le couvert de l'anonymat. Le supérieur des Sulpiciens, Étienne Montgolfier, s'était plaint de La Gazette au gouverneur Haldimand. Et il avait écrit une lettre le même jour à Mgr Briand, pour lui demander qu'on censurât le journal de Mesplet (Dictionnaire biographique... iv, 588). Le père Well, jésuite, avait pour sa part souventes fois répliqué au voltairianisme de $L a$ Gazette sous le pseudonyme de "L'Anonyme». Enfin, «Le Sincère moderne» ${ }^{7} y$ avait fait paraître un article où il suggérait à Mesplet d'abandonner son «papier périodique» et de se consacrer uniquement à l'imprimerie (De Lagrave 1985, 154). Ces interventions faites en catimini témoignent de la faiblesse du clergé: eût-il pu intervenir publiquement et fortement, qu'il l'aurait sans doute fait sans hésiter.

Faisons le point. II ne semble pas y avoir, à proprement parler, d'histoire de la censure au Québec, avant le début du $19^{\mathrm{e}}$ siècle, dans la mesure où quelques cas ne suffisent pas à instaurer des pratiques, des stratégies, des actions concertées ${ }^{8}$. Par contre, notons une étude peu connue et qui, si elle ne fait pas davantage une histoire de la censure, propose une lecture des $17^{\mathrm{e}}$ et $18^{\mathrm{e}}$ siècles qui peut servir de propédeutique au $19^{\text {e }}$ siècle. En effet, dans The Rise of Ecclesiastical Control in Quebec, Walter Alexander Riddell tente de cerner «the sociological and historical factors which led up to and made possible the control of the Roman Catholic Church in Québec.» (Riddell 1916, 5). Riddell démontre que, sous le régime français et jusqu'à la fin du $18^{\mathrm{e}}$ siècle, un ensemble de facteurs (travail, langue, religion, éducation, etc.) modèlent la population "as clay in the hands of the ecclesiastical potter.» (Riddell 1916, 94). Selon Riddell, l'Acte de Québec (1774) mais surtout l'Acte constitutionnel $(1791)^{9}$ accordent au clergé une position culminante et un statut affermi qui lui permettront d'asseoir définitivement son contrôle.

Cette affirmation est sans doute vraie; mais elle devra attendre le milieu du $19^{\circ}$ siècle pour s'ancrer dans la réalité. Car, avant que de maîtriser les paroles divergentes, le clergé devra subir une série d'épreuves accablantes, mais riches d'enseignements, entre 1800 et 1840 .

\section{La censure impraticable (1800-1840)}

Ce qui caractérise le début du $19^{\mathrm{e}}$ siècle, jusqu'en 1840, c'est la faiblesse du clergé face au contrôle de l'imprimé ${ }^{10}$. Trois épisodes en témoignent: la saisie du journal Le Canadien (1810), les pamphlets de l'abbé Chaboillez et les activités d'imprimerie illicites de l'abbé Pigeon. Regardons brièvement ces moments difficiles pour le clergé, en n'oubliant pas toutefois qu'il en tirera toutes les leçons nécessaires.

Les circonstances qui ont entraîné la naissance du Canadien, en 1806, sont bien connues: la Constitution de 1791 qui obligeait les Canadiens à être informés de la chose politique, la naissance de la liberté de la presse ${ }^{11}$ et la nécessité de montrer au public les agissements des gouvernants; enfin, les attaques du journal anglophone The Quebec Mercury (fondé en 1805), particulièrement par Anglicanus, ont conduit les Canadiens, sous l'impulsion de Pierre Bédard, à se doter de leur premier journal.

"Les éditeurs d'un papier libre ne doivent pas craindre de se faire des ennemis.» (D.P. 1807, 141): voilà bien ce quiarrivera au Canadien durantla première phase de son existence. Le gouverneur Craig craint de plus en plus que ce journal ne sème l'agitation. Le prétexte sera, comme pour le journal de Jautard et Mesplet, un article jugé séditieux, paru le 14 mars 1810. Craig fait saisir Le Canadien le 17 mars, et ses propriétaires sont emprisonnés.

Et le clergé, dans cette équipée? Craig demande à Mgr Plessis de lire en chaire sa proclamation expliquant la saisie du Canadien. Mgr Plessis sait bien que "c'est de sa [le gouvernement anglais] protection que dépend la liberté du culte dans la province.» (Mgr Plessis... 19271928, 273), si bien qu'il donne pour consigne à ses curés de lire ladite proclamation $^{12}$. L'appui donné à Craig n'était certes pas pour déplaire à $\mathrm{Mgr}$ Plessis qui, après avoir lu la proclamation le 21 mars, fera ensuite un sermon sur le sujet le $1^{\text {er }}$ avril. Mgr Plessis n'aimait pas la Constitution de $1791^{13}$, pas plus qu'il ne prisait Le Canadien quiaura «constamment été mauvais, soit sous un rapport, soit sous un autre, depuis qu'il est au monde.» (Mgr Plessis... 1928-1929, 180). Cependant, ce qui frappe le plus dans cet épisode, c'est combien le clergé, qui désapprouvait privément Le Canadien, doit s'abriter sous le parapluie gouvernemental pour exercer sa censure. Signe de faiblesse? Sans doute, ce que nous démontrent par ailleurs les épisodes suivants, où la contestation provient des rangs mêmes du clergé ${ }^{14}$.

«L'affaire Chaboillez», bien connue dans I'histoire de l'Église canadienne, doit être versée au dossier de la censure. Un

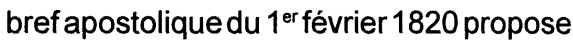
Mgr Lartigue au gouvernement spirituel du district de Montréal. Cette nomination choque particulièrement les Sulpiciens, qui y voient une intervention extérieure dans leurs propres affaires. Cet affront engendrera une série de quatre pamphlets, d'une violence parfois inouïe, signés par des prêtres.

L'abbé Augustin Chaboillez, curé de Longueuil, tire le premier coup, avec Questions sur le gouvernement ecclésiastique du District de Montréal (Chaboillez 1823), où il demande que l'on s'oppose «par tous les moyens que de droit» à cette nomination. La guerre des pamphlets s'amorce! Un jeune avocat de 
24 ans, Pierre Hospice Bédard (fils de Pierre Bédard) répond vertement au curé trop fougueux (Bédard 1823); mais, en vérité, Pierre Hospice Bédard n'est ici qu'un prête-nom, le texte ayant été rédigé par Mgr Lartigue lui-même... Ensuite, Louis-Marie Cadieux fait paraître ses Observations surun écritintitulé "Questions sur le gouvemement ecclésiastique du District de Montréal» (Par un prêtre... 1823). Enfin, Chaboillez revient à la charge, invectivant Bédard (mais il sait que c'est son évêque qui a rédigé le deuxième pamphlet), l'injuriant presque (Réponse de ... 1824).

«Personne au Canada n'avait vu jusque là pareil mépris de l'autorité du pape et des évêques», affirme l'abbé Allaire (t. $6,173)$. Les forces répressives du clergé sont nettement dépassées par l'ampleur de cette contestation qui, de surcroît, vient de l'intérieur. L'élaboration de stratégies censoriales s'imposera bientôt; l'abbé Pigeon en convaincra davantage le clergé, si cela est encore nécessaire.

Curé de Saint-Philippe, l'abbé Pigeon s'oppose au démembrement de sa paroisse, demandé par Mgr Lartigue en septembre 1822. S'ensuit une correspondance acerbe entre Pigeon et «son» évêque qu'il ne reconnaît pas; lui non plus, pour le gouvernement spirituel de Montréal. Plus encore, Pigeon fonde une imprimerie en 1823, sans même chercher l'approbation de l'évêque de Québec. Ce qui en sort a par ailleurs de quoi inquiéter: un journal non approuvé par l'évêque, L'Ecclésiastique, mais surtout les lettres acrimonieuses échangées entre Pigeon et Lartigue autour de toutes ces questions ${ }^{15}$. Encore une fois, les querelles intestines sont lancées dans le public et, démuni, le clergé essaiera de laisser s'éteindre cette contestation.

Cependant, le clergé aura beaucoup appris de ces pénibles événements! Mgr Lartigue n'aura de cesse de demander à l'évêque de Québec une presse et un journal: «les difficultés présentes, [écrit-il], montrent la nécessité d'avoir une presse et unjoumalecclésiastique [...]» (Mgr Lartigue 1942-1943, 127). Et le mot-clef est lancé, celui du pouvoir: le journal représenterait pour les évêques «une arme qui aurait pu leur assurer un grand pouvoir.» (Mgr Lartigue 1942-1943, 132).
Ce pouvoir, cependant, Mgr Lartigue ne l'aura jamais, puisque son évêque ne partage pas cette visée. Le diocèse de Montréal est constitué en évêché en 1836: Mgr Lartigue a alors les coudées franches, mais il est assailli par d'autres préoccupations et surtout, il se fait vieillissant. II reviendra à celui qui a été son secrétaire depuis le début des années 20 et témoin de toutes ces attaques contre le clergé et des réactions d'impuissance; à celui qui partageait les rêves de Mgr Lartigue; à Mgr Bourget, donc, d'instituer en pouvoir ce qui aura été chez Lartigue savoir et vouloir.

\section{La censure intensive-extensive I (1840-1892)}

Avant d'aborder la troisième phase, comprenons ce que veulent dire en l'occurrence intensive et extensive. Les réalités derrière ces concepts sont fort simples. Par censure intensive, on entendra ici toute activité censoriale forte, voire brutale àl'occasion; elle se caractérise alors par la mise en place de mécanismes, d'institutions de contrôle afin de réprimer toute manifestation hétérodoxe. Par censure extensive, on désignera toute régulation du discours social qui est, le plus souvent, le prolongement d'une censure intensive. Charles Grivel nomme endoxa cette censure intériorisée, socialement tout autant qu'individuellement: "La vraie censure est donc le code idéologique considéré comme langage de contrainte du sujet et exprimant ce sujet[...]» (Grivel 1974, 102) En résumé, l'on a le cadre ci-dessous:
Or, la période qui nous intéresse ici (1840-1892) se déploie selon ces phases. D'une part, la censure intensive correspond au règne de Mgr Bourget (1840-1876). Bien sûr, l'on s'efforce de produire l'orthodoxie par divers moyens: le journal Mélanges religieux, la fondation de "L'Oeuvre des bons livres», le Concile provincial de 1854, etc. Mais la répression de la parole hétérodoxe domine tout ce demi-siècle: I'Institut canadien, Le Pays, les pamphlets de Louis-Antoine Dessaulles en sont les victimes fréquentes. Ce concert de censure intensive produit une endoxa étouffant à la source les propos divergents, particulièrement entre 1876 et 1892 . L'espace ne me permet ici que de rappeler ces principaux faits qui, par ailleurs, sont assez bien connus.

Signalons d'abord la série d'initiatives de censure intensive d'incitation que lance Mgr Bourget. La fondation des Mélanges religieu $x^{19}$, en 1840, représente la réalisation d'un rêve de Mgr Lartigue, un journal qui veut «montrer à toutes les classes de la Société la vertu comme la route du bonheur; encourager le bien, de quelque part qu'il vienne; censurer le mal avecl'accent de la charité, dans l'espoirde le guérir [...]»(Prospectus). Puis, l'établissement de l'Oeuvre des bons livres (1844), qui se donne une structure très précise et rigide concernant la constitution des bibliothèques et la diffusion des livres ${ }^{20}$, le tout assorti d'indulgences ${ }^{21}$. Enfin, le 28 mai 1854 s'ouvre le second Concile provincial de Québec, moment capital du progrès de la censure. Jamais auparavant n'avaientété cernés avec autant de fermeté

\section{Types de censure}

1. Censure intensive

vise à réprimer la parole hétérodoxe

2. Censure extensive

cherche à ne faire produire que la parole orthodoxe impositiond'une endoxa Programmation Intériorisation ${ }^{18}$

Effets

\section{Moyens}


tous les aspects dangereux de l'imprimé. Feuilletons, livres immoraux, bibliothèques, bibles falsifiées, instituts littéraires, tout est dénoncé: «Les mauvais livres et les mauvais journaux étant aujourd'hui le grand moyen employé par l'enfer pour perdre les âmes, le curé doit élever souvent la voix pour en inspirer une vive horreur à son peuple.» (Règlement... t. 2, 469).

Toutefois, les Canadiens français ne sont pas tous des bénis oui-oui. L'un de ceux qui, rugissant contre l'ultramontanisme ${ }^{22}$, obligera le clergé à écraser l'hétérodoxie est un laïc ${ }^{23}$, Louis-Antoine Dessaulles $^{24}$, appuyé par la cohorte de libéraux radicaux regroupés autour de I'Institut canadien de Montréal.

Cet Institut fut fondé en $1844^{25}$. Tribune des Rouges ${ }^{26}$ (libéralisme radical), I'Institut canadien commence à inquiéter dès la fin des années 40 , ce qui entraîne plus tard Mgr Bourget à demander une purge de sa bibliothèque. Cette requête engendre une discussionau sein de l'Institut mais, surtout, courrouce Dessaulles, qui somme en vain l'évêque de Montréal de lui indiquer quels livres doivent être retranchés. Mgr Bourget poursuit son offensive contre la presse libérale, qu'il dénonce en 1858 , et particulièrement contre le journal Le Pays, organe des Rouges, qu'il cherche à "empêcher de séduire les fidèles confiés à nos soins". II est impossible de retracer toutes les attaques et contre-attaques qui se succéderont entre 1854 et 1874 : correspondance acerbe entre Le Pays et Mgr Bourget ${ }^{27}$, mise à l'Index par Rome des Annuaires de l'Institut pour 1868 et 1869 , série de pamphlets de Dessaulles, dont particulièrement L'Index (suite de l'Annuaire de 1869) qui déconstruit les stratégies de censure, La Grande Guerre ecclésiastique (1873), victime d'une interdiction locale, et enfin, la célèbre "Affaire Guibord», bien connue, certes, mais qui n'est qu'un épisode des multiples répressions ${ }^{28}$ de l'hétérodoxie entre 1858 et $1874^{29}$.

Ces quelque trente-cinq années spectaculaires du règne de Mgr Bourget culminent, paradoxalement, dans la relative tranquillité des quinze années suivantes, c'est-à-dire jusqu'en $1892^{30}$. En effet, les interventions épiscopales concernant les imprimés locaux viseront surtout les écrits dirigés contre l'Université Laval. On ne reprendra pas ici toute cette question $^{31}$. Contentons-nous de signaler que Elzéar Paquin, médecin, fait paraître La Conscience catholique outragée [... $]^{\beta 2}$, où il accuse Mgr Taschereau et Mgr Fabre d'abandonner la doctrine de Mgr Bourget et d'appuyer le libéralisme qui sévit à l'Université Laval; Mgr Fabre condamne cet opuscule de même que toute récidive, le 13 mars 1882. Quant à l'abbé Alexis Pelletier, il fait paraître sur cette même question un mémoire à tirage réduit, qui à nouveau accuse Mgr Taschereau d'être la principale cause de la division du clergé. En 1884, ce pamphlet tombe entre les mains des autorités épiscopales qui le condamnent officiellement ${ }^{33}$. C'est donc surtout à Québec que surgissent quelques problèmes et, à Montréal, rien de comparable àl'agitation des années 18501876 ne se produit: la censure extensive prescrit, "pré-scrit» pourrait-on dire, ce qui peut être livré publiquement. Cependant, cette ère relativement calme se terminera brutalement en 1892.

\section{La censure intensive-extensive II (1892-1940)}

Montréal déclenche à nouveau les hostilités: les temps forts de la censure intensive sont désignés par l'affaire judiciaire de Canada-Revue, la mise à l'Index du Clergé canadien, sa mission, son oeuvre de Laurent Olivier David et par le zèle intempestif de MgrBruchési, évêque de Montréal ${ }^{34}$. Quant à la censure extensive visant à produire l'orthodoxie au moyen d'institutions d'encadrement de la parole, elle se déploiera surtout à Québec, autour de l'Action sociale, mais aussi à Montréal avecl'Assocation catholique de lajeunesse canadienne-française. Résumons encore une fois les principales étapes de ce dernier volet.

La saga judiciaire de Canada-Revue a déjà fait l'objet d'études approfondies ${ }^{35}$. Ce journal, qui s'était donné pour mission de secouer la société canadiennefrançaise en prônant «la nécessité d'une éducation scientifique et appliquée, le double enseignement, une université laïque et des bibliothèques volantes» (Jetté 1972, 135), devait s'attirer les foudres du clergé. La Semaine religieuse de Montréal contre-attaque et Mgr Fabre condamne le journal, si bien que celui-ci est acculé à la faillite à la suite de pertes financières énormes. Canada-Revue intente un procès à Mgr Fabre: l'issue glorifiera Mgr Fabre et tout le clergé canadien-français.

II n'en demeure pas moins que cette fin de siècle marquera une recrudescence, sinon du libéralisme radical, du moins d'un anticléricalisme violent ${ }^{36}$. Passons ici sur les acrimonieuses Ruines cléricales, attribuées à Aristide Filiatreault ${ }^{37}$, sur le caustique Curés et Bedeaux ${ }^{38}$, pour parler plutôt du seul livre qui ait été mis à l'index par Rome (en plus de deux Annuaires de l'Institut canadien de 1868 et 1869): Le Clergé canadien, sa mission, son oeuvre, de Laurent-Olivier David $^{39}$.

Des élections fédérales sont prévues pour le 23 juin 1896. Wilfrid Laurier est le candidat libéral, et sa politique envers les écoles francophones du Manitoba manque de fermeté, selon l'épiscopat québécois. II faut donc que Laurier soit battu, si bien que les évêques du Québec se prononcent contre le parti libéral un mois avant les élections. Laurier est néanmoins élu et décide de protester, à Rome, contre l'immixion du clergé dans les affaires politiques. Par l'entremise de Gustave Drolet, il accepte que LaurentOlivier David, qui préparait une étude sur la politique canadienne, en tire un pamphlet qui pourra servir de factum à Rome ${ }^{40}$. David accepte, sans savoir toutefois que son opuscule sera au centre d'un débat véhément et que, le 18 décembre, son ouvrage sera mis à l'Index romain. Ce geste permettra aux évêques de condamner localement, le 27 décembre 1896, le journal L'Électeur, qui avait fait paraître par tranches le livre de David ${ }^{41}$.

Finalement, par où cerner le zèle intempestif du successeur de Mgr Fabre, Mgr Paul Bruchési ${ }^{42}$, pour comprendre sa censure répressive? L'évêque de Montréal surveillera lui-même les écrits qui doivent lui être soumis pour censure préalable, il fondera un comité de surveillance, voire un comité de censure pour le théâtre; il se montrera préoccupé par les livres qui franchissent la douane, au point d'écrire à Wilfrid Laurier lui-même; il s'adressera à de nombreuses reprises aux propriétaires de La Presse, de La Patrie, pour les admonester sur le contenu scabreux des faits divers qu'ils relatent; enfin, 
publiquement, il interdira le journal Les Débats (Mandement du 29 septembre 1903), La Semaine (27 juillet 1909), La Lumière (3 juin 1912), Le Pays ${ }^{43}$ (3 juin 1912 et 25 septembre 1913). Les romans ne seront pas épargnés: Marie Calumet de Rodolphe Girard ${ }^{44}$ (1904), "Les Foins» (1909), épisode de la future Scouine (1918) d'Albert Laberge ${ }^{45}$, enfin Le Débutant d'Arsène Bessette (1914) ${ }^{46}$ seront matraqués par Mgr Bruchési.

De 1892 jusque vers 1914 donc $^{47}$, le clergé se voit obligé d'user de mesures répressives pour contrer une série de discours divergents; cependant naît à Québec, en 1907, l'Action sociale catholique. Ce mouvement servira de fer de lance pourétablir une censure extensive remarquable. Le seul coup d'éclat du clergé sera, en 1934, l'interdiction des Demicivilisés, de Jean-Charles Harvey ${ }^{48}$ et une réprobation de L'Ordre, d'Olivar Asselin ${ }^{49}$. Les années 1915-1940 ne marquent pas le déclin de la censure ecclésiastique, mais bien son apogée, auquel aura concouru "l'action positive», dont j'évoquerai la naissance et le rôle pour terminer ce parcours.

Deux mouvements d'action catholique marquent le début du siècle: l'Association catholique de la jeunesse canadienne-française (ACJC) et l'Action sociale catholique (ASC). L'ACJC ne manque pas d'intérêt. Active surtout dans la région montréalaise, orientée vers la jeunesse, cette association, fondée le 13 mars $1904^{50}$, joue un rôle important, avec ses «cercles» dans les collèges, sa revue Le Semeur, ses diverses publications et son «Prix d'action intellectuelle», lancé en 1919. L'Action sociale catholique, cependant, semble encore plus imposante en rayonnement.

La naissance de l'ASC n'est pas sans lien avec ce qui a précédé; en fait, ce mouvement et particulièrement le projet d'un journal «remontait à une dizaine d'années en arrière, au plus fort de la lutte entre l'Épiscopat et les journaux libéraux, L'Électeurnotamment» ${ }^{51}$ etl'interdit contre Laurent-Olivier David. Fondée en 1907, l'ASC vise "l'organisation de toute une campagne de propagande par le livre, par la revue, par le joumal, parle bulletin, par la brochure, parle tract, parles publications de toutes sortes qui peuvent contribuer à la diffusion des connaissances et des idées chrétiennes» ${ }^{52}$.

À cet égard, l'oeuvre principale sera le journal L'Action catholique, appuyé par une imprimerie et une librairie. En 1917, I'ASC lance son Almanach et poursuivra son action éditoriale, sous l'étiquette "L'Action sociale», de manière régulière et intense: près d'une centaine de livres et de brochures paraîtront sous sa responsabilité entre 1907 et 1919.

L'ACJC et l'ASC, auxquelles se conjugueront les efforts du Devoir, de l'Action française ${ }^{53}$ et d'autres relais de la bonne parole ${ }^{54}$, mouleront les générations des années 1910-1940 pour créer un climat de censure extensive formidable: l'âge d'or du clergé ne se situe pas au $19^{e}$ siècle, mais bien durant la première moitié du $20^{e 55}$. Mais c'est aussi, par voie de conséquence, l'âge d'or de la censure extensive. L'harmonie est presque totale; et, en matière de censure, c'est précisément de l'harmonie qu'il faut se méfier, celle-ci pouvant être un autre nom pour le conditionnement et la programmation. Robert Lahaise $(1994,76)$ parle d'un clergé triomphaliste: ce clergé "se retrouve surtous les fronts et récupère tous les mouvements». Cela n'empêche pas toutefois l'émergence de quelques éditeurs laïcs qui provoquent une certaine émancipation de la littérature au début des années 30: Albert Lévesque et Albert Pelletier dominent cette période, le premier avec sa collection «Les romanciers de la jeune génération» de même que ses auteures poétiques avantgardistes (Jovette Bernier, Éva Senécal, entre autres) et le second, pour avoir édité Les Demi-civilisés de Harvey et la poésie détonnante de Medjé Vézina ${ }^{56}$.

\section{Pour conclure}

Je rappellerai que, par ce bref parcours de la censure religieuse au Québec, je n'ai eu d'autre but que de dresser un état de nos connaissances historiques et bibliographiques. Certes, l'ampleur et la complexité du sujet sont les coupables de liens parfois lâches entre les étapes de ce parcours. Par exemple, je n'ai pas parlé des femmes et de leur rapport à la censure. Pourtant, Henriette Dessaulles, Jovette Bernier, Medjé Vézina, Éva Senécal et, même, la Laure Conan
d'Angéline de Montbrun, ont produit des oeuvres dissidentes ou dissonantes, auxquelles s'ajoute l'apport de la presse féminine. Cependant, elles n'ont pas été condamnées, mais occultées ${ }^{57}$.

Mais on peut tout de même espérer que cet aperçu démontre non seulement la quantité de recherches qui demeure à faire, mais aussi l'importance du sujet dans la compréhension de l'histoire culturelle et plus particulièrement littéraire du Québec. On n'a encore qu'une vague idée de l'importance qu'a eue la censure dans la formation des mentalités, des oeuvres, des genres ${ }^{58}$. II ne semble cependant pas exagéré de dire que, vu la force du clergé entre 1840 et 1940 , les activités censoriales que l'on peut attribuer, tant sur le plan de la répression que de l'action positive, ont désigné l'aspect principal de la formation du littéraire: «par plus d'un demi-siècle d'efforts, les censeurs ont pu aseptiserle milieu littéraire et culturel au point de le rendre presque stérile» ${ }^{59}$. La vérité de cette assertion reste à démontrer, et j'espère avoir ici posé les bornes d'une telle recherche, tant sur le plan des limites que des possibilités.

Au milieu du $20^{\circ}$ siècle, c'est tout ce monde qui bascule et qui annonce la fin du contrôle clérical; voilà une autre question, complexe à souhait, que l'antagonisme entre le pouvoir religieux et le pouvoir laïc ${ }^{60}$ durant les années 50 , puis la prééminénce de ce dernier à partir des années 60 . II faut comprendre la difficulté de saisir ce nouveau pouvoir multiforme, qui s'exerce par les relais judiciaire et législatif, le contrôle des subventions, les lois du marché traduites en morale conformiste, la concentration de la presse, etc. Au pouvoir clérical somme toute assez net et clair où la censure était reconnue comme légitime, a succédé un pouvoir diffus qui, explicitement, affirme ne censurer que le minimum nécessaire mais qui, par tout un réseau de ramifications souterraines, échappe au regard et à la conscience. C'est dire la difficulté croissante de l'étude de la censure, des années 40 à nos jours. 


\section{Notes}

1. Le seul article qui tente un survol général de la censure est, de Claude-Marie Gagnon, «La censure au Québec.» Voix \& Images 9, $\mathrm{n}^{\circ} 1$ (automne 1983):103-117. Quant aux rapports entre l'Église et la censure, notons Séraphin Marion, Les Lettres canadiennes d'autrefois, tome VIII: "Littérateurs et moralistes du Canada français d'autrefois», (Hull:Éd. L'Éclair; Ottawa:Éd. del'Université d'Ottawa, 1954), 191 p. L'on y trouve des chapitres importants sur le Tartuffe et Mgr de Saint-Vallier, l'Institut canadien de Montréal et la saga de Canada-Revue. Ajoutons la synthèse de Jean Laflamme et Rémy Tourangeau, L'Église et le théâtre au Québec (Montréal: Fides, 1979), 356 p. R. de Marmande, In Le Cléricalisme au Canada (Paris: Librairie critique Émile Nourry, 1911), 204 p. consacre d'importants chapitres à la littérature et à la liberté de la presse. Signalons enfin un mémoire qui porte nommément sur notre sujet, par Danielle Rainville, Le Monde de l'imprimé et l'Église au Québec, 18801960 (M.A. (Bibl.), Université de Montréal, 1983), $137 \mathrm{p}$. Fondé surtout sur les documents officiels, ce mémoire offre un intéressant parcours de la question.

2. Sans doute m'est-il permis de dire que j'y travaille actuellement, avec la collaboration de Patrick Nicol, du Groupe de recherche sur l'édition littéraire (GRELQ) de l'Université de Sherbrooke.

3. J'exclus ici tout ce qui touche l'autocensure, sur laquelle nous disposons par ailleurs de très peu d'études. De telles recherches appellent de longues et minutieuses enquêtes où la correspondance d'auteur est souvent le filon indispensable. Richard Giguère aborde cette question dans "Alfred DesRochers etla critique cléricale de son temps. Censure et autocensure de L'Offrande aux vierges folles» (1928), dans La Faculté des Lettres. Recherches récentes surl'épistolaire français et québécois, (Département d'Études françaises, Université de Montréal, 1993), p. 163-181.

4. J'aborderai plus en détail ces notions lorsque, dans cette troisième partie, je devrai y recourir.

5. Cette année 1913 marque la dernière condamnation officielle et importante de Mgr Bruchési, en l'occurence contre Le Pays.

6. Une exception cependant, dans le secteur du théâtre toujours : la réaction du clergé à l'endroit du Théâtre de société, "le seul exemple pendant toute la période [17651825] d'une dispute littéraire suivie et publique,» note Baudouin Burger, L'Activité théâtrale au Québec, 1765-1825 (Montréal: Éditions Parti pris, 1974), p. 289. On ne manquera pas de consulter, sur cette «dispute littéraire» qui eut lieu de novembre 1789 à juin 1790, Lorraine Camerlain, Trois interventions du clergé dans l'histoire du théâtre à Montréal: 1789-1790, 1859 et 1872-74 (M.A. (Études littéraires), Université de Montréal, 1979), 186 p. Le titre signale manifestement que deux autres cas du siècle suivant sont analysés, l'ensemble du mémoire tendant à relativiser l'impact qu'eut le clergé sur la vie théâtrale. J'abandonne ici le genre théâtral traité en détails par les études que je viens de signaler, mais non sans noter un mémoire sur un secteur de la vie sociale qui englobe le théâtre: le loisir. Ghislain Labbé signe une étude qui a une incidence directe sur notre sujet, $L$ 'Église, le loisir et la censure au Québec, avant 1960 (M.A. (Sciences du loisir), 1983), $145 \mathrm{p}$

7. J'ai cependant tenté de démontrer que, contrairement à l'opinion reçue à ce sujet, ce journal est essentiellement le produit de Jautart et Mesplet eux-mêmes, sous le couvert d'une trentaine de pseudonymes différents. Voir Jacques Cotnam et Pierre Hébert, «La Gazette littéraire (1778-1779): notre première oeuvre de fiction?» Voix et Images 59 (hiver 1995)

8. L'utilisation de la confession comme contrôle de la lecture désigne une stratégie importante à l'époque; mais son action ne touche que la sphère du privé et ne rayonne nullement dans l'espace public. Voir François Melançon, «Façonner et surveiller l'intime: lire en Nouvelle-France,» In Discours et pratiques de l'intime, sous la direction de Manon Brunet et de Serge Gagnon (Québec: IQRC, 1993).

9. Son jugement sur l'Acte constitutionnel manque toutefois de nuance.

10. N'allons pas en déduire que tout était calme en ce début de $19^{\circ}$ siècle. UnQuébec qui bougeait, par Jean-Pierre Wallot (Trois-Rivières: Boréal Express, 1973), 345 p. démontre le contraire.

11. Voilà un autre pan qu'il faudrait développer, celui de la liberté de presse. Donnons des pistes: Séraphin Marion, «Liberté, liberté chérie...» In Lettres canadiennes d'autrefois, t. III (Hull: Éd. de l'Éclair; Ottawa: Éd. de l'Université d'Ottawa, 1942), p. 17-73; R. de Marmande, Le Cléricalisme au Canada, chapitre VIII; Louis-Arthur Richard, Des délits de presse (Québec: Le Soleil, 1915), 137 p. Adjutor Rivard,Dela libertéde presse (Québec: Librairie Garneau, 1923), 125 p.; Jean-Paul de Lagrave, "La liberté de la presse," Les Journalistes démocrates au Bas-Canada (1791-1840), p. 95-110.

12. Les réactions diverses des curés suite à cette lecture sont des plus intéressantes, et se trouvent dans le Rapport de l'Archiviste de la province de Québec (désormais $R A P Q$ ), 19321933, 68-69. Voir aussi sur cette question Marcel Trudel, «La servitude de l'Église catholique du Canada français sous le régime français,» le Rapport de la Société canadienne d'histoire de l'Église, 1963, p. 11-33, et JeanPierre Wallot, «The Lower Canadian Clergy and the Reign of Terror (1810),» Rapport de la Société canadienne d'histoire de l'Église, 1973, p. 53-60.

13. Cette constitution "n'a d'autre effet réel que de rendre les administrés insolents envers l'administration", écrira quelques années plus tard Mgr Plessis à l'évêque de Telmesse (Mgr Lartigue), 13 septembre 1823 , In $R A P Q$, 1928-1929, p. 174.

14. J'ai étudié les cas de Chaboillez et de Pigeon dans «Le Clergé et la censure de l'imprimé au Québec: les années décisives (1820-1840).» Voix \& Images 44 (hiver 1990): 181-195. Voir aussi Lucien Lemieux, L'Établissement de la première province ecclésiastique au Canada, 1783-1844 (Montréal: Fides, 1968), p. 67-71 et Gilles Chaussé, Jean-Jacques Lartigue, premier évêque de Montréal (Montréal: Fides, 1980), p. 63-65.

15. Correspondance entre l'évêque de Telmesse et le curé de Saint-Philippe, 1825 [?] et Rapports entre le curé de Saint-Philippe et Monseigneur de Québec, 1826 [?]

16. Recours à l'Index, documents officiels, etc.

17. Contrôle des bibliothèques, des librairies, noyautage des éditeurs, de la critique, etc.

18. Contrairement au niveaux précédents, c'est sur le plan individuel que se joue ici la censure; l'individu en arrive à ne plus dire, voire à ne plus penser certaines choses. Cela est encore plus profond que l'autocensure: ce n'est plus du refus dont il est question, mais del'incapacité même de refuser, c'est-à-dire de penser autrement.

19. Voir Denise Lemieux, «LesMélanges religieux, 1841-1852,» In Fernand Dumont, Jean-Paul Montmigny et Jean Hamelin, directeurs, Les idéologies au Canada français, 1850-1900 (Québec: Presses de l'Université Laval, 1971), p. 63 et suivantes.

20. J'ouvre avec hésitation tout ce dossier des bibliothèques, de la librairie, voire des éditeurs ou imprimeurs-éditeurs: l'influence du pouvoir ecclésiastique en rapport avec les relais de diffusion a été, bien sûr, déterminant, et seul l'espace de cet article interdit de pousser l'analyse. Je pourrais à peine rappeler ici les travaux essentiels sur ces questions (Drolet, Lamonde, Gallichan, etc.). Je crois utile de signaler, pourtant, dans L'Évolution du rôle social de l'imprimé et de ses agents aux Québec, "Conférences Aegidius-Fauteux», série 1979-1980: Jean-Louis Roy, «Une région culturelle mal connue: le pouvoir des libraires ou les libraires au pouvoir,» p. 23-45, et Marcel Lajeunesse, «Les bibliothèques québécoises: les avatars de leur rôle social à travers les âges,» p. 46-78. Mais on connaît encore bien peu, et bien mal, le rôle réel qu'a joué le clergé à l'endroit des relais de diffusion du livre.

21. Voir «Mandements d'installation de l'Oeuvre des bons livres à Montréal,» 20 septembre 1845, In Mandements, lettres pastorales, circulaires et autres documents, publiés dans le diocèse de Montréal depuis son érection (désormais MÉM), t. 1, p. 304-312. Voir Olivier Maurault, «L'Oeuvre des bons livres,» Marges d'histoire, tome III: Saint-Sulpice (Montréal: Librairie d'Action canadienne- 
française, 1930), p. 55-104. Sur ce même sujet mais aussi sur les autres initiatives des Sulpiciens au $19^{\mathrm{e}}$ siècle, voirl'étude fouillée de Marcel Lajeunesse, Les Sulpiciens et la vie culturelle à Montréal au XIXe siècle (Montréal: Fides, 1982), 280 p., de même que, sur le Cabinet de lecture paroissial, Pierre Rajotte, Les Mots du pouvoir ou le pouvoir des mots. Essai d'analyse des stratégies ultramontaines au XIX ${ }^{e}$ siècle (Montréal: Hexagone, 1991) en particulier «La nécessité de la censure,» p. $155-158$.

22. Sur l'ultramontanisme et les fondements du pouvoir religieux, voirNadia F. Eid, Le Clergéet le pouvoir politique au Québec. Une analyse de l'idéologie ultramontaine au milieu du XIXe siècle (Montréal: Hurtubise/HMH, 1978), $318 \mathrm{p}$.

23. Je dis un laïc: caril faut consulterà ce sujet Le Laïc dans l'Église canadienne-française de 1830 à nosjours (Montréal: Fides, 1972), 223 p.

24. Signalons l'étude d'Yvan Lamonde, LouisAntoine Dessaules, 1818-1895. Un seigneur libéral et anti-clérical (Montréal: Fides, 1994), $372 \mathrm{p}$.

25. Il est inutile de rappeler cethistorique bien connu. Voir entre autres Théophile Hudon, L'Institut canadien de Montréal et l'Affaire Guibord (Montréal:Beauchemin, 1938), 173 p.; Nancy S. Robertson, The Institut Canadien. An Essay in Cultural History (M.A.(Histoire), University of Western Ontario, 1965), 187 p.; Léon Pouliot, Mgr Bourgetet sontemps. Tome IV: Affrontement avecl'Institut canadien, 1858-1870(Montréal: ÉditionsBellarmin, 1976), 160p. YvanLamonde, Gens de parole. Conférences publiques, essais et débats à l'Institut canadien de Montréal, 1845-1871 (Montréal:Boréal, 1990), 177p.

26. Signalons évidemment l'ouvrage de Jean-Paul Bernard, Les Rouges. Libéralisme, nationalisme et anticléricalisme au milieu du XIX $X^{e}$ siècle (Montréal:PUQ, 1971), 394 p.

27. Des extraits de cette correspondance sont donnés dans «Des documents cruciaux de débat libéralultramontain: les lettres (1862) de MgrBourget aujournal Le Pays,» par Yvan Lamonde et Pierre Nolin, Littératures, 3(1989), 115-204.

28. Multiples répressions auxquelles il conviendrait d'ajouter, autourdel'enseignementdes classiques, la condamnation de brochures de Georges SaintAimé [l'abbéAlexis Pelletier], LettreàMonsieur Baillargeon, évêque de Tloa, sur laquestiondes classiques [...] et Réponse auxdernièresattaques dirigées par Monsieur l'abbé Chandonnet [...] (MEQ, 12 août 1868), t. 4,p. 646. Voir Thomas Charland, «Ungaumiste canadien:l'abbé Alexis Pelletier,» Revue d'histoire de l'Amérique française (désormais RHAF)(septembre 1947): 195. Cetépisode etla question dugaumisme sontt bien étudiés dans Histoire du catholicisme québécois, dirigé par Nive Voisine, Réveil et consolidation. Tome 2, 1840-1898, par Philippe Sylvain etNive Voisine(Montréal:Boréal, 1991), p. 178-187. Biensûr, mêmesijela signale icipour la première fois, l'Histoire du catholicisme québécois est fondamentale pour comprendre la censure ecclésiastique.

29. Sur Guibord, voir aussi Robert Hébert, Le Procès Guibordoul interprétationdesrestes (Montréal: Triptyque, 1992), 196p.

30. Iln'est pas inutile de noter que cette ère de force cléricale (1876-1892) correspond dans son début avec «la victoire totale pour l'Église qui règne dorénavant sur le monde de l'éducation.» (Michel Verrette, L'Alphabétisation au Québec 1660-1900, Ph. D. (Faculté des lettres), Université Laval, 1989, p. 118). La seule remise en question, ajoute Verrette, est due au gouvernement de Félix-Gabriel Marchand, en 1897; ce sera du point de vue de la censure également un moment intense. L'histoire de lacensure, en grande partie, semble suivrel'histoire de l'éducation.

31. Voir AndréLavallé, Québeccontre Montréal. La querelle universitaire, 1876-1891 (Montréal: PUM, 1974), 259p.

32. La Conscience catholique outragée et les droits de l'intelligence violés, par les deux principaux défenseurs de l'Université Laval, Sa Grâce Monseigneur Taschereau, archevêque de Québec, et Sa Grandeur Monseigneur Fabre, évêque de Montréal. Ouvrage réservé pour le public canadien et Notre très Saint Père le Pape (Montréal, 1882).

33. Signalons quelques autres réprobations sans lendemain: Le Monde (1881) et la Revue canadienne (1882), de même que l'interdiction contre la réédition de LaLanterne d'ArthurBuies (1886). L'Index a parfois des répercussions juridiques: le procès contre Cadieux\& Derome au sujet des oeuvres de Victor Hugo doit être signalé ici (voir «Les livresàl'Index,» Le Propagateur 1 (1890-1891): 133-136.

34. L'espace nenous permet pas de parler d'Honoré Beaugrand; mais pour se faire une idée de la manière dont un catholique rigoureuxle considère, voir Thomas Chapais, «Questions de morale littéraire,» In Mélanges de polémique et d'études religieuses(Québec:L'Événement, 1905),p. 125142; Séraphin Marion, "Censure québécoise d'aujourd' huietd'autrefois,» Nouvelle-France 16-17 (mars-juin 1961):52-56.

35. En particulier, voir Pierre Jetté, Le Journal "Canada-Revue» et Mgr Édouard-Charles Fabre (M.A. (Histoire), Université McGill, 1972), 144 p. + Appendices. Aussi: Séraphin Marion, "Canada-Revue et Mgr Fabre," In Les Lettres canadiennes d'autrefois, t. VIII: "Littérateurs etmoralistes du Canada français d'autrefois,»p. 76-96; Jean de Bonville, «La liberté de presse à la fin du XIX ${ }^{\mathrm{e}}$ siècle: le cas de Canada-Revue,» Revue d'histoire de l'Amérique française 31 (mars 1978): 501523; et surtout La Grande Cause ecclésiastique. Le Canada-Revue contre Mgr E. C. Fabre. Procédure, preuve, pièces du dossier, plaidoyers des avocats, reproduction des textes originaux et des notes sténographiques officielles (Montréal: John Lovell \& Son, 1894), 350 p. Enfin, Claude Élizabeth Perreault, «Paul Marc Sauvalle, un journaliste rebelle,» La Petite revue de philosophie 8 (automne 1986): 163-189 (Sauvalle était rédacteur en chef de Canada-Revue en 1892 et 1893).

36. Sur le libéralisme au tournant du siècle, voir l'étude de Fernande Roy, Progrès, harmonie, liberté. Le libéralisme et les milieux d'affaires francophonesà Montréal au tournant du siècle (Montréal:Boréal, 1988), 301 p.

37. Au pays des ruines. I - Ruines cléricales (Montréal: A. Filiatreaultéditeur, 1893), $183 \mathrm{p}$. Notons quel'ouvrage parutmême en traduction anglaise la même année. Filiatreault était «directeur-gérant» de Canada-Revue.

38. Le cléricalisme au Canada. I. Curés et bedeaux (Montréal, 1896), 87 p. Il s'agit d'une charge intempestive contre le clergé. La presse politico-cléricale, par exemple, organise «une boucherie des ames», transformant "toute une population réputée intelligente, en un ridicule essaim de bedeaux et de sacristains branlants.» (p. 33). Parurent la même année sous l'anonymat Saintes Comédies. Le cléricalisme au Canada. II (Montréal) et Les Hommes noirs. Propagande anti-cléricale, Première série quisont de la même eau fielleuse.

39. (Montréal: Eusèbe Sénécal etFils, Imprimeurs, 1896), $123 \mathrm{p}$.

40. Sur les rapports entre Rome et le Canada, l'étude très fouillée de Róberto Perrin, Rome et le Canada. La bureaucratie vaticane et la question nationale, 1870-1903 (Montréal: Boréal, 1993), 345 p., est essentielle.

41. David s'était vu répliquer par P. Bernard (pseudonyme du Père Dominique Ceslas Gonthier), Un manifeste libéral. M. L.-O. David et le clergé canadien (Québec: Léger Brousseau, 1896), 177 p.; Deuxième partie: les écoles du Manitoba (Québec: Léger Brouseau, 1896), 213 p. + Appendices. Voir Thomas Charland, «Les débuts du P. Gonthier dans la polémique,» RHAF, VI (1953): 234246 et, du même, Le Père Gonthier et les écoles du Manitoba. Mission secrète à Rome (1896-1897) (Montréal: Fides, 1979), 131 p. Aussi, Roland Houde, «DominiqueCeslas Gonthier, o.p., 1853-1917. Perception et réception,» La Petite Revue de philosophie 8 (automne 1986): 192-214.

42. Lise Saint-Jacques a produit sur Mgr Bruchési un excellent mémoire qui, en plus de décrire les activités de l'évêque, offre un encadrement théorique des plus utiles: Mgr Bruchési et le contrôle des paroles divergentes: journalisme, polémiques et censures (1896-1910) (M.A. (Histoire), Université du Québec à Montréal, 1987), $140 \mathrm{p}$.

43. Voir l'étude fouillée de Patrice Dutil, Devil's Advocate. Godfroy Langlois and the Politics of Liberal Progressivism in Laurier's Québec (Montréal/Toronto: Robert Davies Publishing, 1994), 376 p. 
44. Voir Marie-Claude Waymel, Dissidence idéologique et dissonance romanesque (le roman québécois de 1900 à 1920 (M.A. (Études littéraires), Université du Québec à Montréal, 1980), 135 p., et Madeleine Charlebois-Dirschauer, Rodolph Girard (1879-1956), sa vie, son oeuvre (Montréal: Fides, 1896), $159 \mathrm{p}$.

45. Voir Annie Alexandre, Le pouvoir comme lecteur: la censure devant "Madame Bovary" et "La Scouine» (Ph.D. (Etudes françaises), Université de Montréal, 1982), 245 p. Le chapitre «Le discours censeur,» p. 187-223 propose des réflexions pertinentes sur la force des paroles qui censurent. La censure de Laberge, c'est cependant celle de l'«oubli»: André Marquis, «Les fluctuations du capital symbolique. De l'oubli et de l'institution," In Robert Giroux et Jean-Marc Lemelin, Le Spectacle de la littérature (Montréal: Tryptique, 1984), p. 107-117.

46. Voir Normand Saint-Pierre, La Censure $d u$ roman "Le Débutant" (1914) de Arsène Bessette: le texte et l'institution (M. A. (Études littéraires), Université du Québec à Montréal, 1984), $242 \mathrm{p}$. Ce roman n'a fait l'objet d'aucune déclaration officielle de Mgr Bruchési.

47. Pouruneréflexion sur les tensions qui marquent le tournant du siècle, on pourra lire «Orthodoxie et hétérodoxie littéraires: le cas du Québec vers 1900,» de Jane Everett, Littératures 1 (1988), p. 91-124.

48. Voir Jean-Charles Harvey, Les Demi-civilisés, édition critique préparée par Guildo Rousseau (Montréal, 1988), particulièrement «Le scandale des Demi-civilisés," p. 34-43.

49. Un dépouillement complet des Mandements de Montréal et de Québec, qu'il serait trop long de faire ici, donne pour la période 19151939 , le plus petit nombre d'interdictions proportionnellement aux périodes antérieures, c'est-à-dire près de trois fois moins.

50. Laurier Renaud, La Fondation de l'A.C.J.C. L'Histoire d'une jeunesse nationaliste (Jonquière, 1972), 154 p. et «La fondation de l'A.C.J.C," In Fernand Dumont et al., Idéologies au Canada français 1900-1929 (Québec: PUL, 1974), p. 173-191.

51. Thomas Charland, "L'interdit du Père Gonthier, O.P., " RHAQ, VIII (1954): 72.

52. Lettre pastorale de Mgr Louis-Nazaire Bégin, Archevêque de Québec, sur l'Action sociale catholique et en particulier sur l'Oeuvre de la presse catholique dans l'Action sociale catholique et l'Oeuvre de la presse catholique. Motifs-programme-organisation-ressources (Québec: Imprimerie Ed. Marcotte, 1907)

53. On pourra consulter, sur l'action éditoriale de l'Action française, mon étude: "Quand éditer, c'était agir. La Bibliothèque de l'Action française (1918-1927),» RHAF 46 (automne 1992): 219-244.

54. «Le Devoir etl'Action sociale, les nationalistes de l'A.C.J.C. tendaient à se rejoindre pour englober toute la jeunesse étudiante dans un vaste mouvement à la fois nationaliste et catholique,》 Robert Rumilly, Histoire de la Province de Québec. XV. Mgr Bruchési (Montréal: Bernard Valiquette, s.d.), p. 57.
On pourrait parler longuement aussi de l'École sociale populaire et de L'Oeuvre des Tracts, par exemple, de même que de l'auto-édition comme seule façon de contrer l'alignement idéologique de la plupart des éditeurs, surtout entre 1915 et 1930

55. Notons que c'est durant cette époque que se tient un discours structuré sur l'Index, discours essentiellement de légitimation: J.-E. Laberge, Index, lectures et morale évangélique (Québec: L'Événement, 1914), 31 p.; Raymond M. Charland, l'Index (Ottawa: Éd. du Lévrier,1938), 190p.; et, un peu plus tard, Édouard Gagnon, Lacensure des livres(Québec: 1945), $223 \mathrm{p}$.

56. Voir L'Édition littéraire en quête d'autonomie. Albert Lévesque et son temps, sous la dir. de Jacques Michon (Sainte-Foy, 1994), 214 p.; dans cet ouvrage, sur Albert Pelletier voir Liette Bergeron, «Les Éditions du Totem (1933-1938),» p. 41-55.

57. Voir Lucie Lequin, «Les Québécoises, une autre révolution?» In Paroles rebelles, sous la dir. de Marguerite Andersen et Christine KleinLataud (Montréal: Éditions du RemueMénage, 1992), p. 219-240.

58. Personne n'a systématiquement abordé l'évolution des genres littéraires au Québec et la censure. Je signale cependant, d'André Carpentier, «Notes en marge d'un historique du fantastique québécois au XIX $X^{*}$ siècle," Voix et Images 55 (automne 1993): 104-120. Dans cette étude, Carpentier pose comme hypothèse que la littérature d'imagination, qui «commençait de s'incarner dans le genre gothique» (p. 120), sera paralysée par l'influence du clergé qui accorde «le primat des valeurs morales sur les valeurs esthétiques» (p. 120). Aussi Joseph Costisella, L'Esprit révolutionnaire dans la littérature canadienne-française (Montréal: Beauchemin, 1968), 316 p. et Yves Dostaler, Les Infortunes du roman dans le Québec $d u$ $X I X^{e}$ siècle (Montréal: Hurtubise/HMH, 1977), particulièrement «Faut-il proscrire le roman?», p. 61-109.

59. Maurice Lemire, Introduction à la littérature québécoise (1900-1939) (Montréal: Fides, 1981), p. 23.

60. Sur la censure religieuse après 1940 , citons à tout le moins «La morale prescrite et vécue au Canada français de l'après-guerre, 19451960,» Cultures du Canada français 8 (automne 1991).

\section{Sources citées}

Allaire, J.-B.-A. Dictionnaire biographique duclergé canadien-français, tome 6, p. 173.

Bédard, Pierre Hospice. 1823. Lettre à M. Chaboillez, Curéde Longueuil, relativementà ses questions sur le gouvernement ecclésiastique du district de Montréal. Montréal: James Lane, $40 \mathrm{p}$.

Bulletin des recherches historiques. 1895. 1,2 section des questions; 1.3 , réponse, p. 45 .

Chaboillez, Augustin (abbé). 1823. Questions sur le gouvernement ecclésiastique du District de Montréal. Montréal: Impr. de Thos. A. Turner, $40 \mathrm{p}$.

\section{P. 1807. Le Canadien 25 juillet: 141}

DeLagrave, Jean-Paul. 1975. Laliberté d'expression en Nouvelle-France, 1608-1760.Montréal:Éd. de Lagrave. $131 \mathrm{p}$

1985. Fleury Mesplet (1734-1794), diffuseur des Lumières au Québec. Montréal: Patenaude, p. 154

Dictionnaire biographique du Canada, tome 4 (1771-1800), p. 588 .

Grivel, Charles. 1974. Les mécanismes de la censure dans le système libéral-bourgeois. La Pensée $\mathrm{n}^{\circ}$ 176(août): 102 .

Imbert, Patrick. 1983. Roman québécois contemporain et clichés. Ottawa: Éd. de l'Université d'Ottawa, p. 154-157.

Jetté,Pierre. 1972.Lejournal Canada-Revue et Mgr Édouard-Charles Fabre, M.A. (Histoire) Université McGill,p. 135

Laflamme, JeanetRémy Tourangeau. 1979.L'Église et le théâtre au Québec. Montréal: Fides, p. 5873.

Lahaise, Robert. 1994. La fin d'un Québec traditionnel. 1: Histoire. Du Canada à «Notre État français». Montréal: L’Hexagone, p. 76

Mandements, lettres pastorales et circulaires des évêques de Québec, tome 1, p. 171-172,303.

MgrLartigue àMgr Panet, 16 décembre 1831. 1942 1943. Rapport de l'archiviste de la province de Québec,p. 127,132.

MgrPlessis à M. François Noiseaux, 22 mars 1810 1927-1928. Rapport de l'archiviste de la province de Québec, p. 273.

Mgr Plessis à Mgr Lartigue, 5 janvier 1824. 19281929. Rapport de l'archiviste de la province de Québec,p. 180.

Parun Prêtre du Diocèse de Québec. 1823 Questions surle gouvernementecclésiastique du District de Montréal. Trois-Rivières:LudgerDuvernay. $32 \mathrm{p}$.

Règlement disciplinaire adopté dans le second concile provincial de Québec. In Mandements, lettres pastorales, circulaires et autres documents publiés, dans le diocèse de Montréal depuis son érection, tome 2, p. 469.

Réponse de Messire Chaboillez, Curéde Longueuil à la lettre de P.H. Bédard; suivie de quelques remarques sur les "Observations» imprimées aux Trois-Rivières. 1824. Montréal:T.A. Turner, $70 \mathrm{p}$.

Riddell, Walter Alexander. 1916. The Rise of Ecclesiastical Control in Québec. New York: Columbia University Press, p. 5, 94.

Roy, Antoine. 1930.Leslettres, lessciencesetles arts au Canada sous le régime français. Paris: Jouve et Cie, éd., p. 84-85, 125-126. 